A. Rossini, F. Borghetti, P. Malcovati, F. Maloberti: "Behavioral Model of Magnetic Sensors for SPICE Simulations"; 12th IEEE Int. Conf. on Electronics, Circuits and Systems, ICECS 2005, Gammarth, 11-14 December 2005, pp. 41-44.

(C)20xx IEEE. Personal use of this material is permitted. However, permission to reprint/republish this material for advertising or promotional purposes or for creating new collective works for resale or redistribution to servers or lists, or to reuse any copyrighted component of this work in other works must be obtained from the IEEE. 


\title{
Behavioral Model of Magnetic Sensors for SPICE Simulations
}

\author{
A. Rossini ${ }^{1}$, F. Borghetti ${ }^{2}$, P. Malcovati ${ }^{2}$, F. Maloberti $^{1}$ \\ ${ }^{1}$ Department of Electronics, University of Pavia, via Ferrata 1, 27100 Pavia, Italy, \\ ${ }^{2}$ Department of Electrical Engineering, University of Pavia, via Ferrata 1, 27100 Pavia, Italy, \\ (andrea.rossini, fausto.borghetti, franco.maloberti, piero.malcovati)@unipv.it
}

\begin{abstract}
This paper describes a general model for magnetic sensors to be used in portable systems. The model, written using the VERILOG-A language, enables the simulation of fully integrated solution. Physics and magnetic features of sensors are accounted for including second order effect like temperature, parasitic capacitance and contact resistance. The behavioral model obtains first magnetization and cycles completely customizable for the hysteresis calculation. The accuracy is very good without requiring heavy computation. The behavioral model describes magneto-resistances, magneto-inductances, fluxgate and Hall devices. The model is verified by computer simulations in the Cadence-Spice environment.
\end{abstract}

\section{INTRODUCTION}

New products perspectives have recently increased the interest for magnetic sensors. Novel developed material, new technique and methods enhance the possibility to effectively sense the magnetic field. Applications for magnetic sensors include the electronic compass, sensors for traffic control, magnet activated switches and magnetic sensor to be used in cellular phone, notebook or handheld device. Other applications are for automotive applications or home appliances: devices based on magnetic field and its measure are used to control the engine or apparatuses used in domestic ambients.

The area addressed by this research is for integrated applications where the features of the magnetic sensor must match the electronic interface that is possibly cointegrated or co-packaged. The key requests are low power and small volume. There are four type of sensors that are suitable for integrated micro-systems: the magneto-resistance, the Hall device, the magnetoinductance and the flux-gate. They change a given physical property when exposed to an external magnetic field. The $d c$ resistance changes in magneto-resistance [1] and Hall devices[2], [3]. The magneto inductance[4] and the flux-gate[5] vary their permeability.

This paper describes the physical quantities involved using equivalent electrical variables, thus permitting the study of the magnetic sensor, its control and the electrical interfacing with a circuit simulator.

\section{TYPE OF SENSORS}

Magnetic sensors can be classified by using the field sensing range. We can distinguish between three categories-low field, medium field, and high field sensing[6]. Magnetic fields less than 1 micro gauss are very low and well below the earth magnetic filed. Sensors with a range of 0.1 gauss to few gauss will be considered Earth's field sensors and sensors with sensitivity above 10 gauss can be considered bias magnet field sensors. Table 1 lists various sensor technologies and shows the corresponding magnetic field sensing ranges. For measuring the Earth magnetic field with sensors that are suitable for portable applications we have the magnetoresistance and magneto-inductance (to be used as discrete sensors). Fluxgates, magneto-resistances require using magnetic material; they, as well as magneto-transistor and Hall sensors can be integrated on CMOS technologies. The use of magnetic material as concentrator can help in increasing their sensitivity. Achieving $2 D$ on chip is possible for fluxgate, Hall with magnetic concentrator, magneto-transistors. By contrast, conventional Hall devices are for $1 D$.

Table 1. Magnetic sensor technology field range.

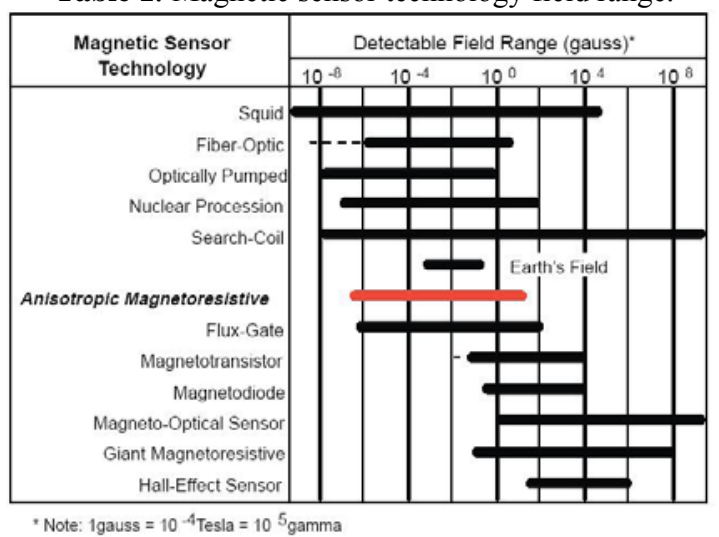

Every sensor has specific characteristics that make it suitable for a given range of applications. In addition to the sensitivity it is necessary to account for the range of temperature, the sensor volume, and its on-chip manufacturability. Magneto-resistances (Fig. 1), Hall devices and flux-gate can be integrated on silicon. 


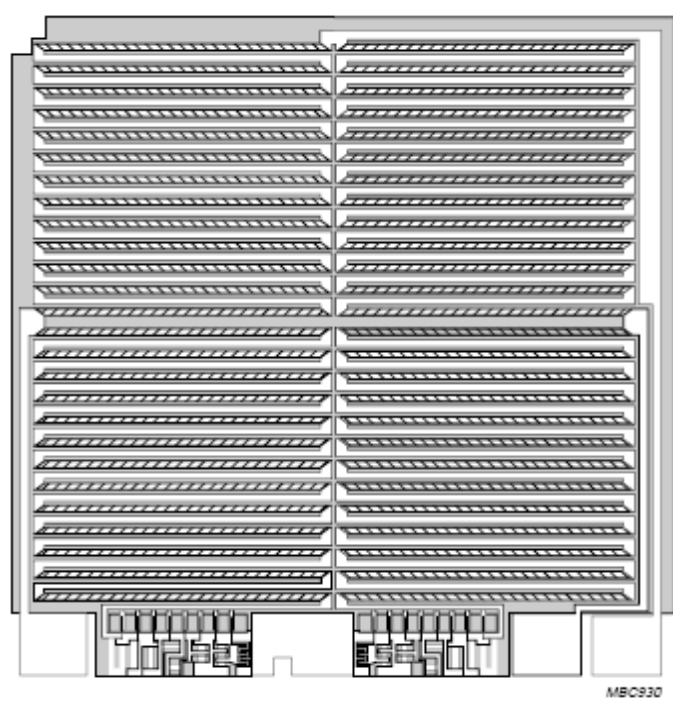

Figure 1. A integrated magneto-resistance.



Figure 2. A magnetic-inductance.

Magnetic-inductances are miniaturizable but they must be used as external components. Fig. 2 show the photo of a commercial product compared with the size of a small coin. The depicted type of sensor is sensitive to the magnetic field in one dimension.

\section{MODELLING THE SENSOR}

The AHDL language code developed for this study describes the behavioral model of four different types of sensors. More specifically, we have used the VERILOG- $A$ language since it is suitable for the $C A D E N C E$ simulation environment widely used for $I C$ design. The symbol of the sensor is a 5 terminal component. The meaning of some of the terminals can change depending of the type of sensor. For all the models we have two main terminals, one of which is the reference, representing the electrical output of the sensor. Thus, the differential voltage across the main terminals or the current through them is the effect of the magnetic field. A voltage generator or a current source through a third terminal represents the input of the device: the external magnetic field, $H$. The remaining two terminals serve for the pick-up coil in flux-gates or for monitoring internal quantities for other sensors.

A set of equation describes the operation of the sensor. The model also includes non-idealities typical of real devices. Performances depend on a number of parameters that are specified in the model card similarly to what is done for active components. The global parameters for a given type of sensor are given in the sensor model card.
The device card contains parameters that are specific for the sensor.

\subsection{Magneto-resistance}

The magneto-resistance changes with external magnetic field, $\mathrm{H}_{\text {ext. }}$. We assume that the output variable is the voltage across the magneto-resistance, $\mathrm{V}_{\text {out }}$. It is given by

$$
V_{\text {out }}=R_{0}\left(1+\alpha \cdot \rho \cdot H_{\text {ext }}\right) \cdot I
$$

where $R_{0}$ is the resistance without external field, $\rho$ is the resistance for unit of length and $\alpha$ is a fitting parameter accounting for the different orientation between magnetization and current flowing in the sensor.

\subsection{Magneto-inductance}

The external magnetic field $H_{\text {ext }}$ changes the value of the magneto-inductance. We use as output variable the voltage across the inductance, $V_{\text {out }}$. It is estimated by using the set of equations

$$
\begin{gathered}
H=n \cdot I+\alpha \cdot H_{\text {ext }} \\
B=B_{\text {sat }} \cdot \tanh (\beta H+\gamma) \\
V_{\text {out }}=n \cdot l \cdot S \cdot \frac{d B}{d t}
\end{gathered}
$$

where $\alpha$ accounts for the possible loss of magnetic field; $\beta=\mu_{0} \mu_{r} / B_{\text {sat }}$ is proportional to the magnetic permeability in vacuum, $\gamma$ is the residual magnetic field due to hysteresis, $n$ is the number of coils for unit of length, $l$ is the length of the sensor and $S$ is the area.

\subsection{Flux-gate}

A given current through a primary coil induces a voltage on the secondary (pick-up) coils that depends on the external magnetic field $H_{\text {ext }}$. The output variable is $V_{\text {out }}$, the pick-up signal. The used equations are

$$
\begin{gathered}
H_{1}=n \cdot I+\alpha \cdot H_{\text {ext }} \\
B_{2}=B_{\text {sat }} \cdot \tanh \left(\beta H_{1}+\gamma\right) \\
V_{\text {out }}=n \cdot l \cdot S \cdot \frac{d B_{2}}{d t}
\end{gathered}
$$

where $H_{1}$ is the magnetic field generated by the primary coils, $B_{2}$ the field on the secondary coils. The tanh function models the magnetization curve.

\subsection{Hall sensor}

The voltage across the Hall terminal depends on the bias current and the external magnetic field $H_{\text {ext }}$. The output variable is the Hall voltage $V_{\text {out }}$. It is estimated by

$$
\begin{gathered}
R_{H}=R_{0}\left(1+\Delta R_{0} \cdot H_{\text {ext }}\right) \\
V_{\text {out }}=R_{H} \cdot I_{\text {bias }}
\end{gathered}
$$

where $R_{H}$ is the Hall resistance, while $\Delta R_{0}$ is the resistivity sensitivity to the magnetic field. 


\section{SECOND ORDER EFFECT}

The main equations given above do not account for a number of second order effects that change the output signal in a linear or a non-linear way.

\subsection{Temperature drift}

The temperature effect is accounted for with a polynomial equation that enables non-linear responses. The polynomial parameters are given in the model card. The effectiveness of the model has been tested using a relaxation oscillator that incorporates a magnetoinductance. Fig. 3 shows the variation with temperature of the frequency of operation for a given constant magnetic field. The result, that matches experimental data, shows a significant temperature dependence. Thus, the electronic interface will be required to correct the drift using the signal from a temperature sensor.

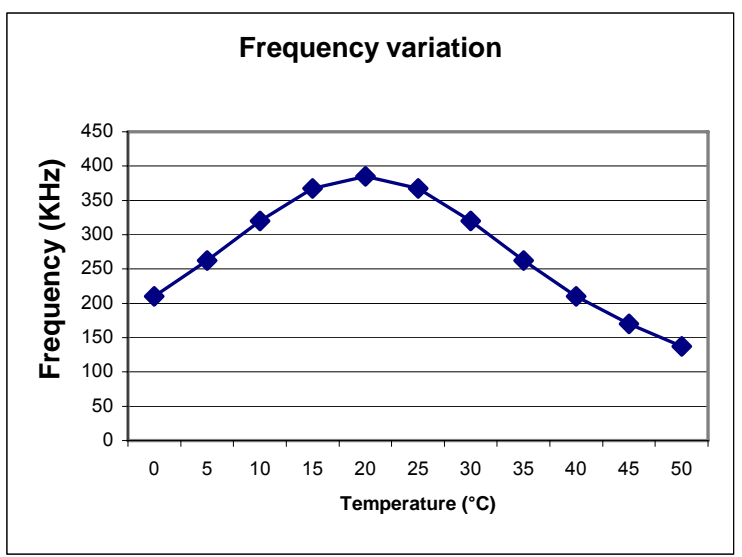

Figure 3. Effect of the temperature over the frequency.

\subsection{Parasitic effect}

The effect of package, solder dot and, more in general, parasitics affect the output response. Their effect is accounted for with an equivalent circuit [7], [8] that includes parasitic capacitance and parasitic contact resistances. Fig. 4 shows the circuit used to account for parasitic effects. Obviously their importance depends on the circuit where the sensor is used. For example, for the relaxation oscillator circuit there is a significant dependence on the parasitic capacitance (Fig. 5) and resistance (Fig. 6).

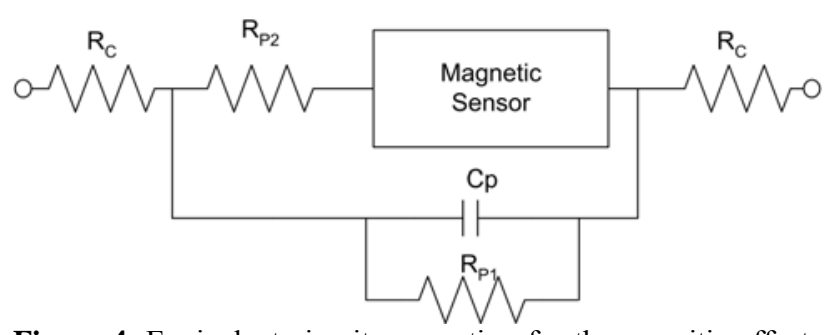

Figure 4. Equivalent circuit accounting for the parasitic effects of the contact $\left(\mathrm{R}_{\mathrm{C}}\right)$ and of the package $\left(\mathrm{R}_{\mathrm{P} 1}, \mathrm{R}_{\mathrm{P} 2}\right.$ and $\left.\mathrm{C}_{\mathrm{P}}\right)$.

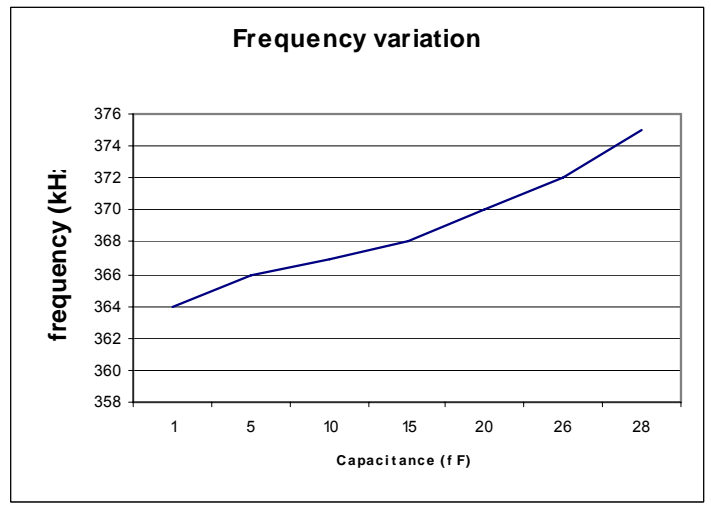

Figure 5. Effect of the temperature over the frequency.

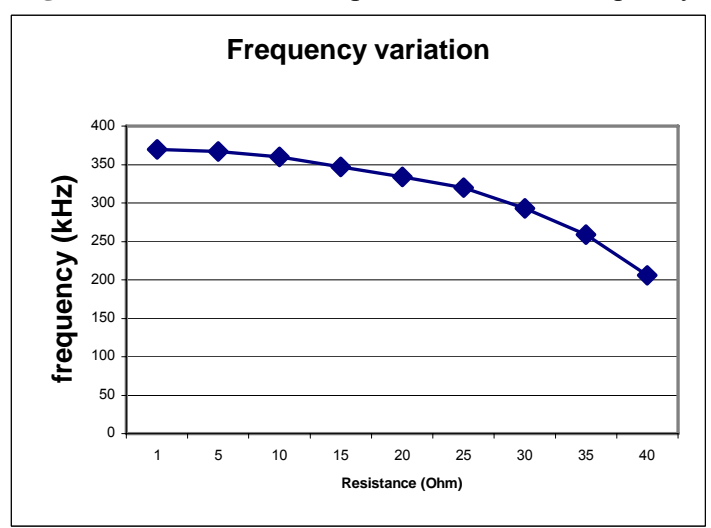

Figure 6. Effect of the resistance over the sensor frequency.

Observe that the effect of the resistance worsens the response of the sensor more than the variation of parasitic capacitance.

\subsection{Dispersed field}

The model enables specifying the amount of magnetic field concatenated by the sensor. A suitable parameter takes into account the loss in magnetic field. The loss diminishes the sensitivity of the sensor and its value controls the overall gain.

\subsection{Hysteresis}

When a magnetic sensor uses magnetic material it is necessary to account for hysteresis. The effect comes from the magnetization of domains that do not completely demagnetize when the magnetic field goes to zero. Moreover, when all the magnetic domains are oriented in the direction of the external field the value of $B$ saturates. The model of the $B-H$ response use equation (10) as primary function. It assumes that $B$ depends as tanh on $H$ but through the parameters $B_{\text {sat }}, \delta, \beta$ permits scaling and shifts. $B_{r e s, i}$, and $H_{\text {res }, i}$ are 0 for the first magnetization cycle.

The $B-H$ function is recalculated every time the magnetic field reaches a given value and reverses. The value reached by the magnetic field, $B_{i n v, i}$, defines a new pair of $B_{r e s, i}$ and $H_{\text {res }, i}$. They are calculated according to equation (11). The use of equation (10) with the pairs $B_{r e s, i}$ and $H_{r e s, i}$, and $-B_{\text {res }, i}$ and $-H_{\text {res }, i}$, defines two primary 
functions. They are suitable for the new $B(H)$ response in the positive or negative quadrants of the $B-H$ plane. The transition between the two functions as $H$ changes its sign is done with a weighting function $W(H)$ that is FermiDirac like.

$$
\begin{gathered}
B_{P}\left(H, B_{R e s, i}, H_{R e s, i}\right)=\delta \cdot B_{s a t} \cdot \tanh \left(\beta H+H_{R e s, i}\right)+B_{R e s, i} \\
B_{R \mathrm{es}, i}=\delta \cdot B_{I n v, i} ; \quad H_{R \mathrm{e} s, i}=\delta \cdot \tanh ^{-1}\left(B_{R \mathrm{e} s, i} / B_{\text {Sat }}\right) \\
B(H)=W(H) \cdot B_{P}\left(H, B_{R e s, i}, H_{R e s, i}\right)+ \\
+[1-W(H)] \cdot B_{P}\left(H,-B_{R e s, i},-H_{R e s, i}\right)
\end{gathered}
$$

$\beta$ takes into account the temperature effect and size of the sensor. The algorithm is light and easy to simulate. Fig. 6 shows simulation results. It represents several cycles starting from the first magnetization curve. The model also enables local hysteresis loops.

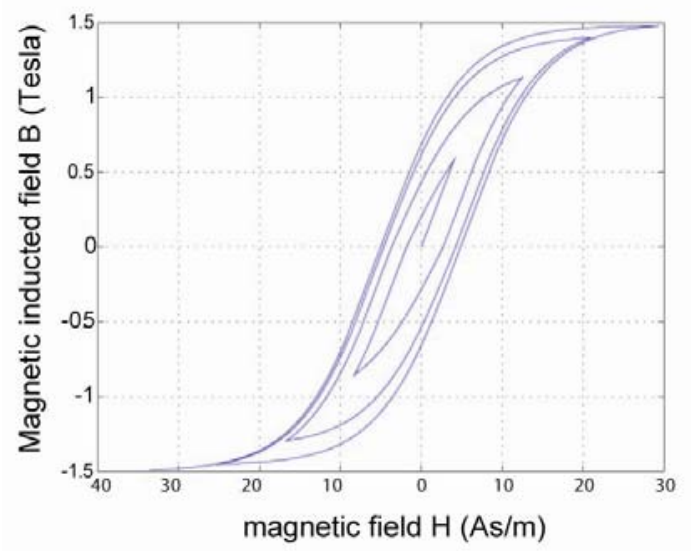

Figure 6. Hysteresis in the curve B-H.

\section{VERIFICATION OF THE MODEL}

The model can be used together with electronic circuits that incorporate the magnetic sensor. A good test-bench is the use of a magneto-inductance sensor in a relaxation oscillator, Fig. 7. The operation of the circuit is as follow: suppose that the comparator output is zero. The output voltage rises at the power on until the voltage across the resistance $R_{2}$ exceeds the threshold $V_{t h l}$; the output of the comparator switches down and the current flows in the opposite direction. The oscillation depends on the time constant of the $R / L$ series and is $(R 1+R 2) / L$.

The circuit has been built with discrete components and a miniaturized magneto inductance. The result of measurement well fit simulations made with the same circuit topology and the parameters provided in the datasheet of the magneto-inductance [4].

Fig.8 compares the experimental and simulation results for different external magnetic fields. The matching is good: the error does not exceed $15-20 \%$ in the entire range of measured field. The modelling of the hysteresis increases the simulation time by $40 \%$ and improves the matching accuracy up to $10 \%$.

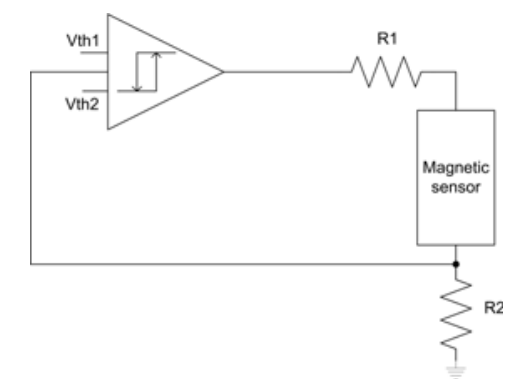

Figure 7. Relaxation oscillator including a magneto-inductance.

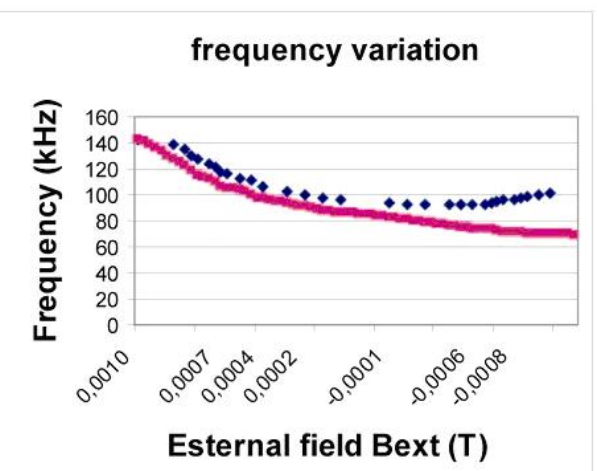

Figure 8. Experimental (continuous line) and simulated results (dotted line).

\section{CONCLUSION}

The proposed $A H D L$ VERILOG-A model is a good tradeoff between complexity and computation load. The model describes the four different type of magnetic sensors with relatively simple equations but can account for second order terms. A behavioral model for studying the hysteresis has been developed and tested. The accuracy of results has been experimentally verified with a $R-L$ relaxation oscillator that uses a magneto-inductance as inductive component. The obtained matching is as good as $10 \%$ thus demonstrating the validity of the used equations.

\section{REFERENCES}

[1] Philips semiconductor, datasheet SC17, 09 Jan 1997

[2] Honeywell, Application Notes AN214 available at www.honeywell.com

[3] Michel Demierre "Improvements of CMOS Hall Microsystems and Application for Absolute Angular Position Measurements" Ph.D. Thesis, Ecole Polytechnicque Federale, Lausanne September 2003.

[4] Application Notes $n^{\circ} 1000083$ R02 available at www.pnicorp.com

[5] M.Schneider "CMOS Magnetotransistor and Fluxgate Vector Sensors", Ph.D. Thesis n. ${ }^{\circ}$ 12746, Physical Electronics Laboratory, ETH Zurich.

[6] Michel Demierre "Improvements of CMOS Hall Microsystems and Application for Absolute Angular Position Measurements" Ph.D. Thesis, Ecole Polytechnicque Federale, Lausanne September 2003.

[7] Martin Ohara, "Modelling non ideal inductors in Spice" Newport Components, Milton Keynes, November 1994.

[8] Z Wang, J. Deen and A. Rahal "Accurate Modelling of Thin-Film Resistor up to $40 \mathrm{GHz}$ ", Proceeding ESSDERC, 24-26 September 2002, pages 307-310. 